\title{
Complex Large-Scale Energy Resource Management Optimization Considering Demand Flexibility
}

\author{
$1^{\text {st }}$ Bruno Canizes \\ GECAD Research Group, Polytechnic of Porto (ISEP/IPP) \\ Porto, Portugal \\ brmrc@isep.ipp.pt \\ $3^{\text {rd }}$ Fernando Lezama \\ GECAD Research Group, Polytechnic of Porto (ISEP/IPP) \\ Porto, Portugal \\ flz@isep.ipp.pt
}

\author{
$2^{\text {nd }}$ João Soares \\ GECAD Research Group, Polytechnic of Porto (ISEP/IPP) \\ Porto, Portugal \\ jan@isep.ipp.pt
}

$4^{\text {th }}$ Zita Vale

Polytechnic of Porto (ISEP/IPP)

Porto, Portugal

zay@isep.ipp.pt

\begin{abstract}
As renewable energy sources penetration is increasing in the power distribution network, an energy aggregator can provide a highly flexible generation and demand as required by the smart grid paradigm. However, this energy aggregator entity needs adequate decision support tools to overcome the complex challenges and deal with a number of energy resources. So, the energy resource management is crucial for the aggregator, to increase the profits, reduce the operation costs, reduce the carbon footprint and also to improve the system stability. Thus, this paper proposes a model for a large-scale energy resource scheduling problem of aggregators in a smart grid. Also, it is compared the performance of five evolutionary algorithms to solve this kind of problem. A realistic case study is performed using a real distribution network in Zaragoza, Spain. The results show that load flexibility can contribute to the profitability improvement of the aggregators' entities.
\end{abstract}

Index Terms-Demand response, differential evolution, distribution network, electric vehicle, evolutionary computation, load flexibility, smart grid.

\section{INTRODUCTION}

The future power systems will require to deal with an even higher number of distributed energy resources (DER) under market conditions. But some issues emerge from this inevitability. Several DER units are not able to participate in the current electricity markets due to their small size; their variability nature (wind and solar units), where the contribution to the grid operation may result in economic penalties as a result of unexpected unbalances; and different ownerships restrains the cooperation and communication between neighbouring units. To mitigate the issues associated with the DER units penetration, the aggregation of those units can be considered. Such aggregation enables the same visibility, controllability,

This work has received funding from Portugal 2020 under SPEAR project (NORTE-01-0247-FEDER-040224) and from National Funds through the FCT Portuguese Foundation for Science and Technology, under Project UIDB/00760/2020. João Soares is supported by FCT CEECIND/02814/2017 grant. and market functionality as conventional generation [1]. A virtual power player (VPP) can be an entity that aggregates several types of energy sources, namely distributed generators (DG) units, and it is responsible for managing them using a set of advanced tools to raise their value and competitiveness. However, VPPs require complex optimization models, control, and secure communications to run properly [2].

To allow efficient and cost-effective operation, energy aggregators, i.e., the VPPs require proper energy resource management (ERM) tools to deal with the increasing number of resources [3]. The day-ahead energy scheduling is an essential part of an ERM system to obtain the expected operation cost (or profit) while providing adequate decisions one day in advance. However, energy scheduling is quite challenging due to the high number of resources. Adopting advanced energy management models is critical for successful implementation of smart grids (SG).

New players as well as energy resources are emerging: large penetration of renewable based generation [4]; demand flexibility [5]; electric vehicles (EV) [6]; energy efficiency measures [7]; building energy management parties [8], among others. The complexity to manage and operate the transmission and distribution power networks, as well as the interactions between the traditional and the new players, has increased due to this new paradigm in the electric power systems. Now, rather than look to the overall picture of overall consumption and generation, the operators of the electric power systems must consider the small resources of new players. Thus, the problem to solve in this domain is now a complex task. So, new approaches have been proposed in the research field to address this complexity. One of the proposed solutions is to consider hierarchical management with the inclusion of increased intelligence at each level of the hierarchical control [9]. To deal with the increased complexity, the development of new algorithms using advanced optimization techniques and 
artificial intelligence are being proposed and tested [10].

In this paper, we have chosen the differential evolution (DE) [11], [12] and two of its variants HyDE and HyDE-DF (due to its success in many applications and easy implementation [13], an improved version of the well-known particle swarm optimization (PSO) [14], and the vortex search (VS) [15] to solve the large-scale scheduling problem in smart grids.

This paper is organized as follows: after this introduction, Section II presents the proposed problem formulation. The case study to show the application of the proposed problem formulation is shown in Section III. The results and its discussion are presented in Section IV. Finally, Section V presents the most relevant conclusions.

\section{Problem Formulation}

A detailed description of the adopted methodology for the day-ahead energy resource management scheduling problem is presented in this section.

The objective function $O C$ (1), represents the expected day-ahead operation costs in monetary units (m.u.), and is minimized over the scheduling horizon $T$. The scheduling time horizon covers the 24 hours of the next day.

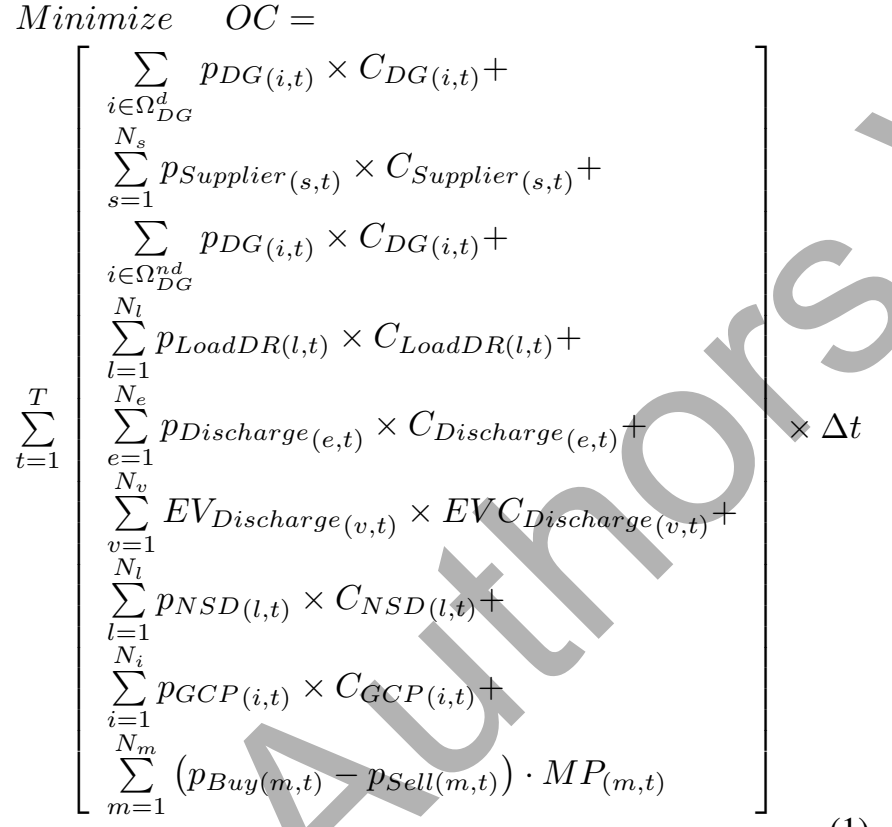

where:

$\Omega_{D G}^{d}$ is the set of dispatchable DG units; $\Omega_{D G}^{n d}$ is the set of non-dispatchable DG units; $i$ is the index of DG units; $s$ is the index of external suppliers (main); $N S$ is the number of external electricity suppliers; $p_{\text {Supplier }}(s, t)$ is the active power scheduled for external supplier $s$ in period $t$ (MW); $C_{\text {Supplier (s,t) }}$ is the costs of the energy supplier $s$ in period $t$ (m.u./MWh); $p_{D G_{(i, t)}}$ is the forecasted non-dispatchable DG unit $i$ in period $t(\mathrm{MW}) ; C_{D G(i, t)}$ is the generation cost of DG unit $i$ in period $t$ (m.u./MWh); $p_{\text {LoadDR(l,t) }}$ is the active power reduction of load $l$ in period $t(\mathrm{MW}) ; C_{L o a d D R(l, t)}$ is the demand flexibility cost of load $l$ in period $t$ (m.u./MWh); $p_{\text {Discharge }(e, t)}$ is the active power discharge of energy storage system (ESS) $e$ in period $t(\mathrm{MW}) ; C_{\text {Discharge }}(e, t)$ is the discharging cost of ESS $e$ in period $t$ (m.u./MWh); $E V_{\text {Discharge }(v, t)}$ is the active power discharge of EV $v$ in period $t(\mathrm{MW}) ; E V C_{\text {Discharge }(v, t)}$ is the discharging cost of EV $v$ in period $t$ (m.u./MWh); $p_{N S D(l, t)}$ is the active power of non-supplied demand of load $l$ in period $t$ (MW); $C_{N S D(l, t)}$ is the non-supplied demand cost of load $l$ in period $t$ (m.u./MWh); $p_{G C P(i, t)}$ is the generation curtailment power of DG unit $i$ in period $t(\mathrm{MW}) ; C_{G C P(i, t)}$ is the curtailment cost of DG unit $i$ in period $t$ (m.u./MWh); $p_{B u y(m, t)}$ is the active power bought in market $m$ in period $t(\mathrm{MW}) ; p_{\operatorname{Sell}(m, t)}$ is the active power sold in market $m$ in period $t(\mathrm{MW}) ; M P_{(m, t)}$ is the market energy price (m.u./MWh); $N_{s}$ is the number of external suppliers; $N_{l}$ is the number of loads; $N_{e}$ is the number of ESS units; $N_{v}$ is the number of EV; $N_{i}$ is the number of generation units; $N_{m}$ is the number of markets.

The power balance constraint (2) is present in the proposed model. The amount of generated energy should equal the amount of consumed energy at every instant $t$.

$$
\begin{aligned}
& \sum_{i \in \Omega_{D G}^{d}} p_{D G(i, t)}+\sum_{s=1}^{N_{s}} p_{\text {Supplier }_{(s, t)}}+ \\
& \sum_{i \in \Omega_{D G}^{n d}}\left(p_{D G(i, t)}-p_{G C P(i, t)}\right)+ \\
& \sum_{l=1}^{N_{l}}\left(p_{N S D(l, t)}+p_{\text {Load } D R(l, t)}-p_{\text {Load }(l, t)}\right)+ \\
& \sum_{v=1}^{N_{v}}\left(E V_{\text {Discharge }_{(v, t)}}-E V_{\text {Charge }_{(v, t)}}\right)+ \\
& \sum_{e=1}^{N_{e}}\left(p_{\operatorname{Discharge}_{(e, t)}}-p_{\text {Charge }_{(e, t)}}\right)- \\
& \sum_{m=1}^{N_{m}}\left(P_{\text {Purchase }(m, t)}-p_{\text {Sell }(m, t)}\right)-\sum_{i \in \Omega_{B}} P_{\text {Inj }(i, t)}=0 \forall t
\end{aligned}
$$

Constraint (3) guarantees the reactive power balance in each distribution network bus.

$$
\begin{aligned}
& \sum_{s=1}^{N_{s}} Q_{\text {Supplier }_{(s, t)}}+\sum_{i \in \Omega_{D G}^{d}} Q_{D G(i, t)}-\sum_{i \in \Omega_{L}^{b}} Q_{\text {Load }(i, t)}- \\
& \sum_{i \in \Omega_{B}} Q_{\text {Inj }(i, t)}=0 \forall t
\end{aligned}
$$

This equation (4) represents the injected active power in each bus of the network.

$$
\begin{aligned}
& P_{I n j(i, t)}=V_{(i, t)} \sum_{j \in \Omega_{B}} V_{(j, t)}\left(G_{(i, j)} \cdot \cos \theta_{(i, j, t)}+\right. \\
& B_{(i, j)} \cdot \sin \theta_{(i, j, t)} \quad \forall i \in \Omega_{B}, \forall(i, j) \in \Omega_{l}
\end{aligned}
$$

The injected reactive power in each bus is represented by the equation (5).

$$
\begin{aligned}
& Q_{I n j(i, t)}=V_{(i, t)} \sum_{j \in \Omega_{B}} V_{(j, t)}\left(G_{(i, j)} \cdot \sin \theta_{(i, j, t)}-\right. \\
& B_{(i, j)} \cdot \cos \theta_{(i, j, t)} \forall i \in \Omega_{B}, \forall(i, j) \in \Omega_{l}
\end{aligned}
$$


The active power flow for each network line is given by the equation (6).

$$
\begin{aligned}
& P_{(i, j, t)}=\left(V_{(i, t)}^{2}-V_{(i, t)} \cdot V_{(j, t)} \cdot \cos \theta_{(i, j, t)}\right) \cdot G_{(i, j, t)}- \\
& \left(V_{(i, t)} \cdot V_{(j, t)} \cdot \operatorname{sen} \theta_{(i, j, t)}\right) \cdot B_{(i, j)} \\
& \forall i \in \Omega_{B}, \forall j \in \Omega_{B}, \forall(i, j) \in \Omega_{l}
\end{aligned}
$$

Equation (7) gives the reactive power flow for each line.

$$
\begin{aligned}
& Q_{(i, j, t)}=-\left(V_{(i, t)}^{2}-V_{(i, t)} \cdot V_{(j, t)} \cdot \cos \theta_{(i, j, t)}\right) \cdot B_{(i, j)}- \\
& \left(V_{(i, t)} \cdot V_{(j, t)} \cdot \operatorname{sen} \theta_{(i, j, t)}\right) \cdot G_{(i, j)} \\
& \forall i \in \Omega_{B}, \forall j \in \Omega_{B}, \forall(i, j) \in \Omega_{l}
\end{aligned}
$$

The apparent power flow equation, as can be seen in equation (8), is given by the square root of the active power flow and reactive power flow squares.

$$
S_{(i, j, t)}=\sqrt{P_{(i, j, t)}^{2}+Q_{(i, j, t)}^{2}} \quad \forall(i, j) \in \Omega_{l}
$$
(9).

The maximum power flow in each line is constrained by

$$
0 \leq S_{(i, j, t)} \leq S_{(i, j)}^{\max } \quad \forall(i, j) \in \Omega_{l}
$$

The voltage magnitude of each bus is constrained by a maximum and minimum deviation (10).

$$
V_{(i)}^{\min } \leq V_{(i, t)} \leq V_{(i)}^{\max } \quad \forall i \in \Omega_{B}
$$

The maximum and minimum angle deviation is constrained by (11).

$$
\theta_{(i)}^{\min } \leq \theta_{(i, t)} \leq \theta_{(i)}^{\max } \quad \forall i \in \Omega_{B}
$$

where:

$\Omega_{B}$ is the set of buses; $\Omega_{l}$ is the set of lines; $P_{I n j(i, t)}$ is the active injected power in bus $i$ for the period $t$ (MW); $Q_{\operatorname{Inj}(i, t)}$ is the reactive injected power in bus $i$ for the period $t$ (Mvar); $Q_{\text {Supplier }(s, t)}$ is the reactive power supplied by substation $s$ in period $t$ (Mvar); $Q_{D G(i, t)}$ is the reactive power supplied by DG $i$ in period $t$ (Mvar); $Q_{\operatorname{Load}(i, t)}$ is the reactive power demand in bus $i$ for the period $t$ (Mvar); $P_{(i, j, t)}$ is the active power flow in the $i, j$ line for period $t(\mathrm{MW}) ; Q_{(i, j, t)}$ is the reactive power flow in the $i, j$ line for period $t$ (Mvar); $S_{(i, j, t)}$ is the apparent power flow in the $i, j$ line for period $t$ (MVA); $V_{(i, t)}$ is the voltage magnitude in the bus $i$ for period $t(\mathrm{~V}) ; \theta_{(i)}$ is the voltage angle in the bus $i$ for period $t(\mathrm{rad}) ; G_{(i, j)}$ is the real term of the element $i, j$ in the bus admittance matrix; $B_{(i, j)}$ is the imaginary term of the element $i, j$ in the bus admittance matrix; $V_{(i)}^{\min }$ is the minimum voltage magnitude limit in the bus $i(\mathrm{~V}) ; V_{(i)}^{\max }$ is the maximum voltage magnitude limit in the bus $i(\mathrm{~V}) ; \theta_{(i)}^{\min }$ is the minimum voltage angle limit in the bus $i(\mathrm{rad}) ; \theta_{(i)}^{\max }$ is the maximum voltage angle limit in the bus $i(\mathrm{rad}) ; S_{(i, j)}{ }^{\max }$ is the maximum admissible line flow between bus $i$ and bus $j$ (MW).

\section{A. Power generation}

A binary variable represents the commitment status of dispatchable DG units. 1 means that the unit is connected and 0 means that the unit is not connected. Maximum and minimum limits for active and reactive power in each period $t$ ((12) and (13), respectively) can be formulated as:

$$
\begin{aligned}
& X_{D G(i, t)} \cdot P_{D G M i n L i m i t}(i, t) \leq \\
& P_{D G(i, t)} \leq X_{D G(i, t)} \cdot P_{D G M a x L i m i t}(i, t) \\
& \forall t, \forall i \in \Omega_{D G}^{d}
\end{aligned}
$$

$$
\begin{aligned}
& X_{D G(i, t)} \cdot Q_{D G M i n L i m i t}(i, t) \leq \\
& Q_{D G(i, t)} \leq X_{D G(i, t)} \cdot Q_{D G M a x L i m i t(i, t)} \\
& \forall t, \forall i \in \Omega_{D G}^{d}
\end{aligned}
$$

The upstream supplier (external supplier / main) maximum limit in each period $t$ regarding active power (14) and reactive power (15) can be formulated as:

$$
\begin{aligned}
& X_{\text {Supplier }(t)} \cdot P_{\text {SMinLimit }(t)} \leq P_{\text {Supplier }(t)} \leq \\
& X_{\text {Supplier }(t)} \cdot P_{\text {SMaxLimit }(t)} \\
& \\
& X_{\text {Supplier }(t)} \cdot Q_{\text {SMinLimit }(t)} \leq Q_{\text {Supplier }(t)} \leq \\
& X_{\text {Supplier }(t)} \cdot Q_{\text {SMaxLimit }(t)} \forall t
\end{aligned}
$$

where:

$X_{D G(i, t)}$ is the binary variable of state of DG unit $i$ in period $t ; P_{D G M i n L i m i t(i, t)}$ is the minimum active power of dispatchable DG unit $i$ in period $t(\mathrm{MW}) ; P_{D G M a x L i m i t}(i, t)$ is the maximum active power of dispatchable DG unit $i$ in period $t(\mathrm{~kW})$.

\section{B. Energy storage systems}

The ESS constraints are shown below. The ESS charge and discharge cannot be simultaneous (16). So, two binary variables guarantee this condition for each ESS:

$$
X_{E S S(e, t)}+Y_{E S S(e, t)} \leq 1 \quad \forall t, \forall e
$$

The maximum battery balance for each ESS can be write as (17):

$$
\begin{aligned}
& E_{\text {Stored }(e, t)}=E_{\text {Stored }(e, t-1)}+\eta_{c(e)} \cdot P_{\text {Charge }_{(e, t)}} \cdot \Delta t \\
& -\frac{1}{\eta_{d(e)}} \cdot P_{\text {Discharge }_{(e, t)}} \cdot \Delta t \quad \forall t, \forall e
\end{aligned}
$$

The maximum and minimum discharge limit for each ESS can be write as (18), (19):

$$
\begin{gathered}
P_{\text {Discharge }_{(e, t)}} \leq P_{\text {DischargeLimit }_{(e, t)}} \cdot X_{E S S(e, t)} \\
\forall t, \forall e \\
P_{\text {Discharge }_{(e, t)}} \geq 0 \quad \forall t, \forall e
\end{gathered}
$$

The maximum and minimum charge limit for each ESS can be formulated as (20), (21):

$$
\begin{aligned}
& P_{\text {Charge }}^{(e, t)} \leq P_{\text {ChargeLimit }}^{(e, t)} \cdot Y_{E S S(e, t)} \quad \forall t, \forall e \\
& P_{C_{\text {harge }}(e, t)} \geq 0 \quad \forall t, \forall e
\end{aligned}
$$


The maximum battery capacity limit for each ESS is write as (22):

$$
E_{\text {Stored }(e, t)} \leq E_{B a t C a p(e)} \quad \forall t, \forall e
$$

Minimum stored energy to be guaranteed at the end of period $t$ can be formulated as (23):

$$
E_{\text {Stored }(e, t)} \geq E_{\text {MinCharge }(e, t)} \quad \forall t, \forall e
$$

where:

$X_{E S S(e, t)}$ is the binary variable representing discharging state of $\operatorname{ESS} e$ in period $t ; Y_{E S S(e, t)}$ is the binary variable representing charging state of $\operatorname{ESS} e$ in period $t ; E_{\text {Stored }(e, t)}$ is the energy stored in ESS $e$ in period $t(\mathrm{MWh}) ; \eta_{c(e)}$ charging efficiency of ESS $e(\%) ; \eta_{d(e)}$ discharging efficiency of ESS $e(\%) ; P_{\text {DischargeLimit }_{(e, t)}}$ maximum active discharge rate of ESS $e$ in period $t(\mathrm{MWh}) ; P_{\text {ChargeLimit }}(e, t)$ maximum active charge rate of ESS $e$ in period $t(\mathrm{MWh}) ; E_{B a t C a p}(e)$ maximum energy stored allowed by ESS $e(\mathrm{MWh}) ; E_{\text {MinCharge }}(e, t)$ minimum energy stored required in ESS $e$ in period $t(\mathrm{MWh})$.

\section{Electric vehicles}

The charge and discharge of each EV is not simultaneous. So, two binary variables are needed for each EV and can be represented by (24):

$$
x_{E V(v, t)}+y_{E V(v, t)} \leq 1 \quad \forall t, \forall v
$$

Battery balance for each EV is represented by (25). The energy consumption for period $t$ travel has to be considered jointly with the energy remaining from the previous period and the charge/discharge occurred in the period:

$$
\begin{aligned}
& E_{\text {Stored }(v, t)}=E_{\text {Stored }(v, t-1)}-E_{\text {Trip }}(v, t) \\
& \eta_{c(v)} \cdot E V_{\text {Charge }_{(v, t)}} \cdot \Delta t-\frac{1}{\eta_{d(v)}} \cdot E V_{\text {Discharge }}(v, t) \\
& \forall t, \forall v
\end{aligned}
$$

When connected to the grid an EV cannot discharge to the grid more than the admissible discharge rate. The discharge limit for each EV considering battery discharge rate can be formulated as (26), (27):

$$
\begin{gathered}
\operatorname{EV}_{\text {Discharge }_{(v, t)}} \leq E V_{\text {DischargeLimit }_{(v, t)}} \cdot x_{E V(v, t)} \\
E V_{\text {Discharge }_{(v, t)}} \geq 0 \quad \forall t, \forall v
\end{gathered}
$$

When connected to the grid the vehicle cannot charge more than the admissible charge rate. The charge limit for each EV considering battery charge rate can be formulated as (28), (29):

$$
\begin{gathered}
E_{\text {Charge }_{(v, t)}} \leq E_{\text {ChargeLimit }_{(v, t)}} \cdot y_{E V(v, t)} \\
E_{\text {Charge }_{(v, t)} \geq 0} \geq 0 \quad \forall t, \forall v
\end{gathered}
$$

The maximum battery capacity limit for each EV can be formulated as (31):

$$
E_{\text {Stored }(v, t)} \leq E_{B a t C a p}(v) \quad \forall t, \forall v
$$

The minimum stored energy must be be guaranteed at the end of period $t$ and is represented by (31):

$$
E_{\text {Stored }(v, t)} \geq E_{M \text { inCharge }_{(v, t)}} \quad \forall t, \forall v
$$

where:

$X_{E V(v, t)}$ is the binary variable representing discharging state of EV $v$ in period $t ; Y_{E V(v, t)}$ is the binary variable representing charging state of EV $v$ in period $t ; E_{\text {Stored }(v, t)}$ is the energy stored in EV $v$ in period $t(\mathrm{MWh}) ; \eta_{c(v)}$ charging efficiency of EV $v(\%) ; \eta_{d(v)}$ discharging efficiency of EV $v$ $(\%) ; E V_{\text {DischargeLimit }}(v, t)$ maximum active discharge rate of $\mathrm{EV} v$ in period $t(\mathrm{MWh}) ; E V_{\left.\text {ChargeLimit }_{(}, t\right)}$ maximum active charge rate of EV $v$ in period $t(\mathrm{MWh}) ; E_{B a t C a p}(v)$ maximum energy stored allowed by $\mathrm{EV} v(\mathrm{MWh}) ; E_{\text {MinCharge }(v, t)}$ minimum energy stored required in $\mathrm{EV} v$ in period $t(\mathrm{MWh})$.

\section{Demand flexibility}

Equation 32 formulates a demand response model, namely direct load control, in which the consumer receives an incentive if their load is reduced. The maximum amount that each load $l$ can be reduced in each period $t$ is repreented by (32):

$$
p_{\text {LoadDR }(l, t)} \leq P_{\text {LoadDRMaxLimit }}(l, t) \quad \forall t, \forall l
$$

where:

$P_{\text {LoadDRMaxLimit }}$ is the maximum limit of active power reduction of load $l$ in period $t(\mathrm{MW})$.

\section{E. Electricity market}

The market offers and bids are limited through (33)-(37), namely maximum and minimum energy sale and purchase, respectively. A market bid cannot coexist with a market offer (sale) at the same time in the same marketplace (37).

$$
\begin{aligned}
& P_{\text {Sell }(m, t)} \leq P_{\text {MarketOfferMax }(m, t)} \cdot X_{\text {Market }(m, t)} \\
& \forall t, \forall m
\end{aligned}
$$

$$
P_{\text {Sell }(m, t)} \geq P_{\text {MarketOfferMin }(m, t)} \cdot X_{\text {Market }(m, t)} \forall t, \forall m
$$

$$
\begin{aligned}
& P_{\text {Purchase }(m, t)} \leq P_{\text {MarketBuyMax }}(m, t) \\
& \forall t, \forall m
\end{aligned}
$$

$$
\begin{aligned}
& P_{B u y(m, t)} \geq P_{\text {MarketBuyMin }(m, t)} \cdot Y_{\text {Market }(m, t)} \\
& \forall t, \forall m
\end{aligned}
$$

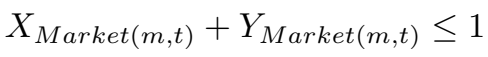

where:

$P_{\text {MarketOfferMax }(m, t)}$ is the maximum energy sale allowed in market $m$ in period $t(\mathrm{MW}) ; P_{\text {MarketOfferMin }(m, t)}$ is the minimum energy sale allowed in market $m$ in period $t$ (MW); $P_{\text {MarketBuyMax }(m, t)}$ is the maximum energy purchase allowed in market $m$ in period $t$ (MW); $P_{\text {MarketBuyMin }(m, t)}$ is the minimum energy purchase allowed in market $m$ in period $t$ (MW); $X_{\text {Market }(m, t)}$ is the binary variable that represents an offer in market $m$ in period $t ; Y_{\operatorname{Market}(m, t)}$ is the binary variable that represents a bid in market $m$ in period $t$. 


\section{CASE STUdY}

The developed model is tested using a case study based on a real distribution network with 201 buses, located in Zaragoza, Spain (Fig. 1) [16]. The original data is slightly modified to include high penetration of DG units, corresponding around $70 \%$ of the total installed capacity power. The installed capacity of cogeneration units represents $33 \%$, the photovoltaic represents about $30 \%$, wind power represents $22 \%$, small hydro represents and biomass units represent $11 \%$ and $4 \%$, respectively. Additionally, $4000 \mathrm{EVs}$ has been considered.

The energy aggregator in this case study is capable of managing 118 DG units, the energy bought from external supplier, 6 ESS units (the charging and discharging efficiency considered for the ESS units is 90\%), $4000 \mathrm{EVs}, 168$ loads points aggregated by bus and 89 aggregated consumers with DR programs (direct load control). In this case study, it is assumed that the aggregator manages the customers in the area, with the goal to minimize the total operation costs. Table I shows the energy data and respective prices. The information of price is shown in monetary units per $\mathrm{kWh}$ (m.u./kWh) and the capacity in MW. The prices have been designed according to [17].

TABLE I: Energy resources characterization of the distribution network.

\begin{tabular}{|c|c|c|c|c|c|}
\hline \multicolumn{2}{|c|}{ Energy resources } & $\begin{array}{c}\text { Prices } \\
\text { (m.u./MWh) } \\
\text { Min-max }\end{array}$ & $\begin{array}{c}\text { Capacity } \\
\text { (MW) } \\
\text { Min-max }\end{array}$ & $\begin{array}{c}\text { Forecast } \\
(\mathbf{M W}) \\
\text { Min-max }\end{array}$ & Units \# \\
\hline Biomass & - & $120-120$ & $0.00-1.45$ & - & 1 \\
\hline CHP & - & $80-100$ & $0.00-4.00$ & - & 4 \\
\hline Small hydro & - & $100-100$ & $0.12-0.35$ & - & 1 \\
\hline Photovoltaic & - & $160-160$ & - & $0.00-1.76$ & 82 \\
\hline Wind & - & $100-100$ & - & $0.52-1.65$ & 30 \\
\hline $\begin{array}{c}\text { External } \\
\text { Supplier }\end{array}$ & - & $100-100$ & $0.00-7.30$ & - & 1 \\
\hline \multirow{2}{*}{ Storage } & Charge & $120-120$ & $0.00-1.50$ & - & 6 \\
\cline { 2 - 7 } & Discharge & $180-180$ & $0.00-1.50$ & - & \\
\hline $\begin{array}{c}\text { Electric } \\
\text { Vehicle }\end{array}$ & Charge & $130-130$ & $0.00-24.80$ & - & 4000 \\
\cline { 2 - 7 } & Discharge & $150-150$ & $0.00-23.12$ & - & \\
\hline $\begin{array}{c}\text { Demand } \\
\text { Response }\end{array}$ & $\begin{array}{c}\text { Reduce } \\
\text { program }\end{array}$ & $70-70$ & $0.27-0.62$ & - & 89 \\
\hline Load & - & $90-150$ & - & $3.94-90.9$ & 168 \\
\hline Market & - & $80-100$ & $0.00-4.00$ & - & 1 \\
\hline
\end{tabular}

Fig. 2, Fig. 3, and Fig. 4 shows the power generation by DG, the demand forecast, and the energy market prices, respectively.

\section{A. Algorithm settings}

In this work, it is compared the performance of five EAs to solve the ERM scheduling optimization problem proposed in Section II, namely the DE [18], the more recently proposed HyDE and HyDE-DF [12], [19], an improved PSO [14], and the Vortex [15]. DE, HyDE, HyDE-DF, and PSO are population-based approaches with very similar mechanisms, performing a number of evaluation functions (EF) equal to the size of the population in each iteration. Vortex is not strictly speaking a population-based method but evaluates for each iteration a given number of neighbour solutions. Thus, results in the same number of EF per iteration as the other algorithms.
1) Particle swarm optimization: Particle swarm optimization belongs to the swarm intelligence class, where the particles (problem solutions) coordinate their actions by modifying their position towards the optimum value. A fitness function improve their position in each iteration [14].

2) Differential evolution: Differential evolution uses a population of individuals to optimize a function. In the initialization stage, solutions are generated randomly within the lower and upper ranges. Differential evolution follows the general iterative of evolutionary algorithm by creating new solutions, applying a mutation and recombination operator, and performing elitist selection, i.e., the better solutions survive for the next generation in each iteration.

3) Vortex search: Vortex search is classified as a singlesolution based metaheuristic. Although, its framework is analogous to the evolutionary algorithms. In each iteration, the number of neighbor solutions are generated using a multivariate Gaussian distribution around the initial solution. The solutions are evaluated in the fitness function, and the singlesolution is updated with the best solution found. The iterative process is repeated until a stop criterion is reached [15].

4) $H y D E$ and HyDE-DF: The hybrid-adaptive differential evolution is a self-adaptive version of DE proposed in [12]. The HyDE uses an operator known as "DE/target-toperturbed $d_{b}$ est/1" that modifies the DE/target-to-best/1 strategy [13], with a perturbation of the best individual, and the self-adaptive mechanism. The hybrid-adaptive differential evolution with decay function [19] is an improved version of HyDE. This new version incorporates a decay function to perform a transition in the iteration process from the main operator of HyDE to the basic operator of DE. Thus, it will be allowed an enhance phase of exploration in the early stage of evolution and stress the exploitation in later stages of the optimization process.

For more detailed explanations of the selected EA, the reader can consult the cited references for further information.

The size of population/neighbour solutions has been set to 5 with 2000 iterations for all the algorithms. For DE, the mutation factor and recombination constant $(F$ and $\mathrm{Cr}$ ) were set to the recommended values 0.5 and 0.9 respectively [18]. HyDE and HyDE-DF [12] are a self-adaptive parameter versions but initial values for $F^{i}$ and $C r$ where set to 0.5 . Vortex search algorithm does not have any parameter to configure [15]. The five algorithms were run for 30 trials each, and the results correspond to the mean value of those 30 trials.

\section{B. Algorithms implementation}

The decision variables of this ERM optimization problem are coded into vector space. The variables for active and reactive power generated by an external supplier and DG are between the limits of the respective generators. Regarding the EV and ESS variables, they are positive if they are charging or negative if they are discharging. For demand flexibility variables, they are coded between the maximum and minimum possible demand reduction (normally the minimum is considered 0 ). The considered binary variables are coded 


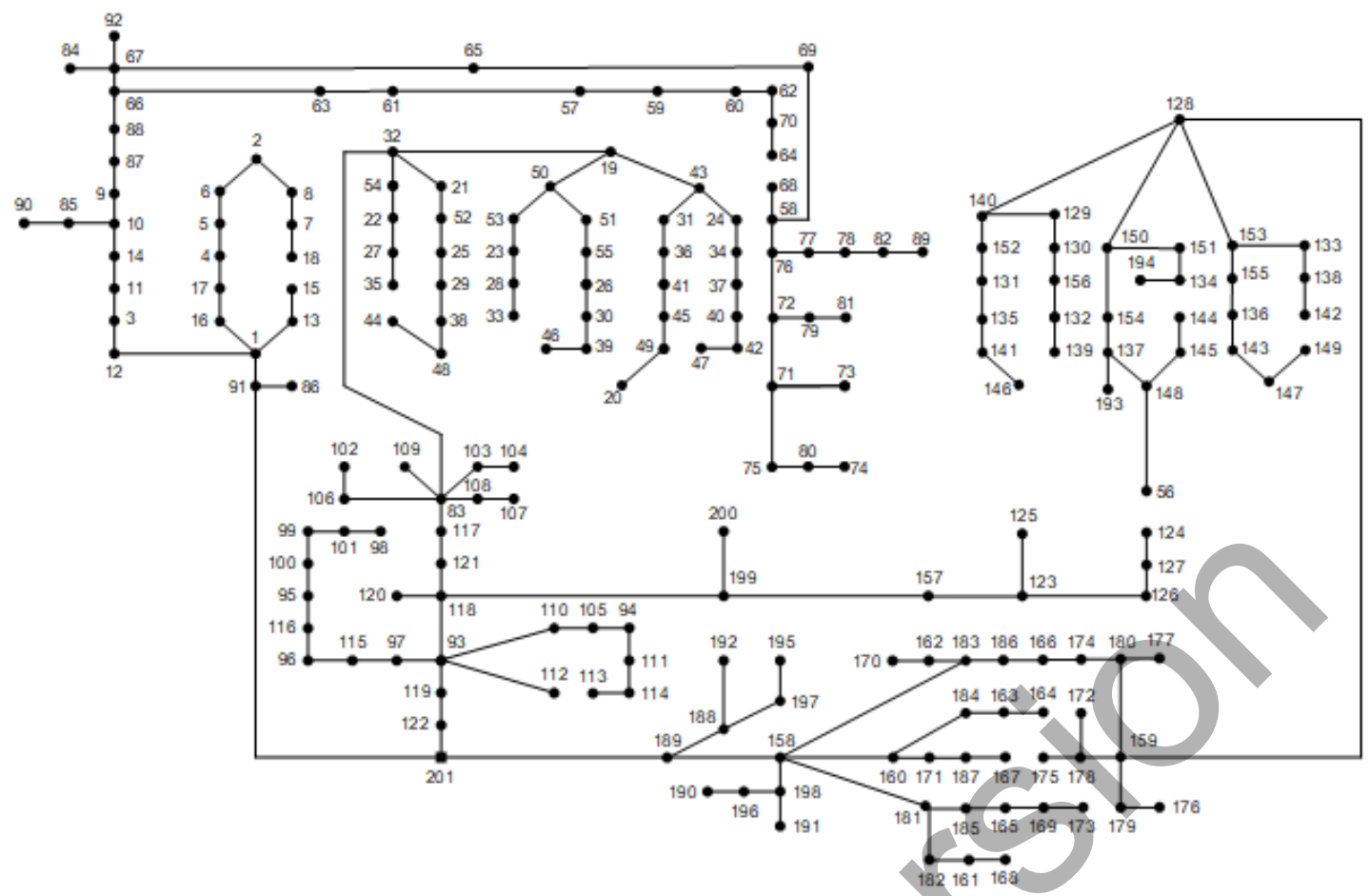

Fig. 1: 201-bus medium voltage distribution network (adapted from [16]).

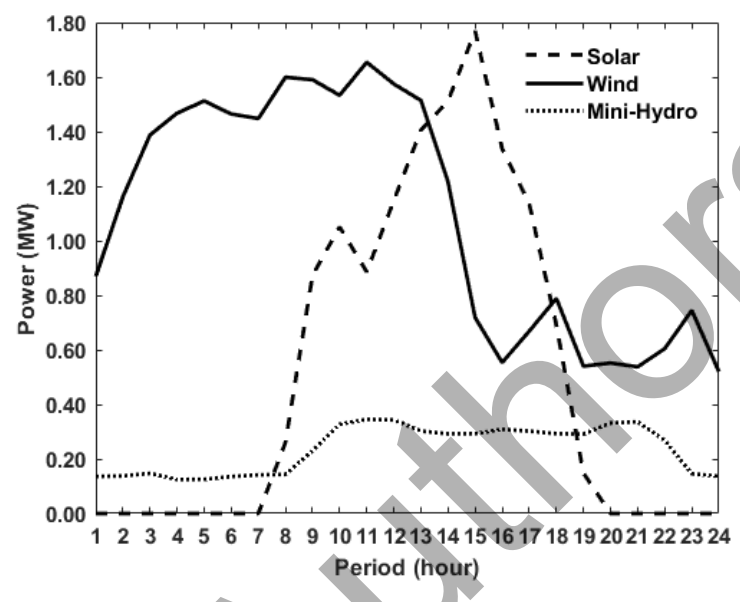

Fig. 2: Distributed power generation forecast. as continuous but using a rounding mechanism (continuous variable between 0 and 1 is rounded to unity).

Additionally, a heuristic mechanism is implemented to predispatch the DG resources with lower prices to improve the metaheuristics' efficiency and effectiveness. With the exception of not dispatchable DGs, the generation units are sorted by merit order according to their costs. The developed heuristic will pre-schedule the DGs units and external supplier acquisition and at the same time balance the generation and the demand in each period, taken into account the generation/demand values for EVs, ESS and demand flexibility variables, which are sought by the metaheuristics internal mechanism.

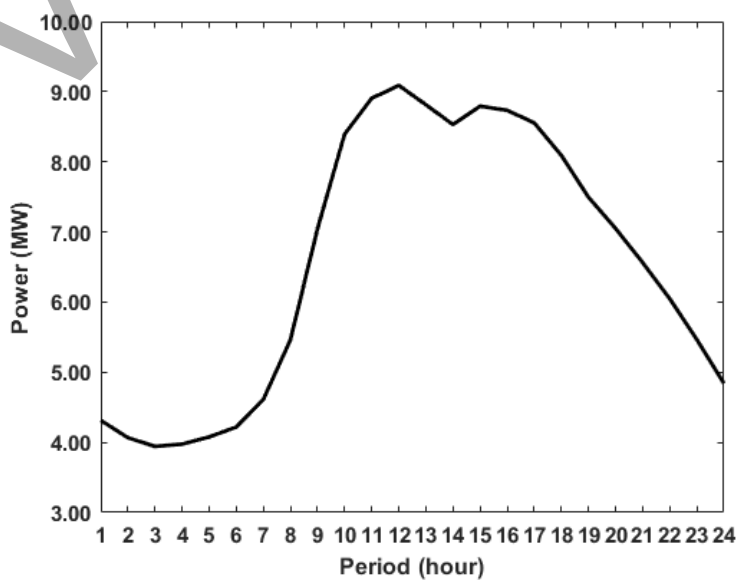

Fig. 3: Load demand forecast.
After this process, a power flow algorithm (based on [20]) is run before the final evaluation (in each iteration) by the metaheuristics process to check the network conditions. Thus, the metaheuristics will have the information to evaluate the technical power network constraints, namely the voltage magnitude and angle limits and thermal limits of the power lines. The identified violations are handled through penalties in the fitness function, and also using direct repair, i.e., the solutions is corrected by changing the variable value, whenever possible, and re-evaluating the objective function without adding further penalties. For instance, if an ESS charged a value that causes a higher amount than the ESS's capacity in a given period, that 


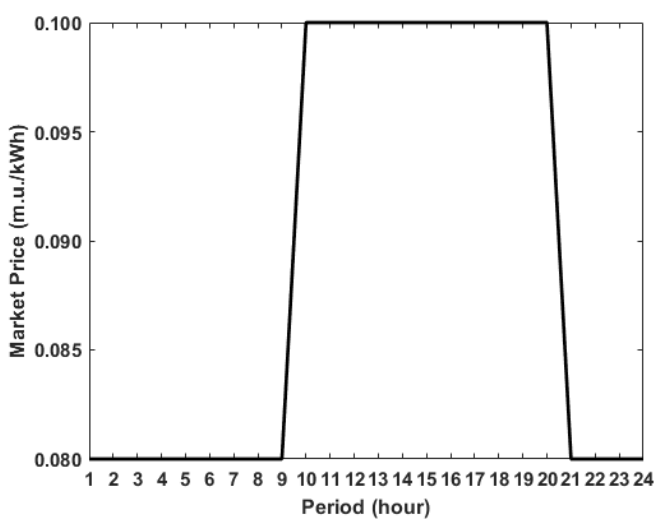

Fig. 4: Market prices.

variable can be directly reduced without causing violation in the capacity.

\section{Results AND Discussion}

The work was developed in a computer with one Intel Xeon E5-2620 v2 processor with 16 GB of RAM running Windows 10 Pro using the MATLAB R2018a. The average values of total operation cost considering and not considering demand flexibility (reference case), the improvement for the aggregator of using the demand flexibility, and the CPU times to perform the 2000 iterations are presented in Table II. It can be seen in this table that the HyDE and HyDE-Df present the best overall costs for the aggregator, which also corresponds to the lower fitness. The differences between these algorithms are small in terms of costs and CPU time. The convergence behaviour of the algorithms in terms of their fitness value evolution considering the demand flexibility and the reference case are depicted in Fig. 5 and Fig. 6, respectively.

TABLE II: Achieved results by the tested evolutionary algorithms.

\begin{tabular}{|c|c|c|c|c|c|c|}
\hline \multirow{2}{*}{$\begin{array}{c}\text { Evolutionary } \\
\text { Algorithm }\end{array}$} & \multicolumn{2}{|c|}{$\begin{array}{c}\text { Overall Costs } \\
\text { (m.u.) }\end{array}$} & \multicolumn{2}{|c|}{ Improvement } & \multicolumn{2}{c|}{$\begin{array}{c}\text { CPU time } \\
\text { (s) }\end{array}$} \\
\cline { 2 - 7 } & With DR & $\begin{array}{c}\text { Without } \\
\text { DR }\end{array}$ & (m.u.) & $(\%)$ & With DR & $\begin{array}{c}\text { Without } \\
\text { DR }\end{array}$ \\
\hline DE & 33072.01 & 33172.31 & 100.29 & 0.30 & 270.71 & 276.29 \\
\hline PSO & 16800.36 & 16874.59 & 74.23 & 0.44 & 280.99 & 288.92 \\
\hline Vortex & 24589.61 & 24617.24 & 27.63 & 0.11 & 335.42 & 315.93 \\
\hline HyDE & 16705.84 & 16866.14 & 160.30 & 0.95 & 500.69 & 527.70 \\
\hline HyDE-DF & 16721.07 & 16866.14 & 145.07 & 0.86 & 326.79 & 312.33 \\
\hline
\end{tabular}

It is possible to see that the curves have practically the same shape for both cases, only that in the reference case (without demand flexibility), the fitness achieved is worst for all the algorithms when compared with the case where demand flexibility is considered. It is also shown that the Vortex algorithm has a slower convergence on the first stage of evolution than the other algorithms. It can also be interesting to check that PSO presents good performance for solving this complex problem of ERM.

The variants HyDE and HyDE-DF present the best performance in terms of overall costs. The reason is that HyDE

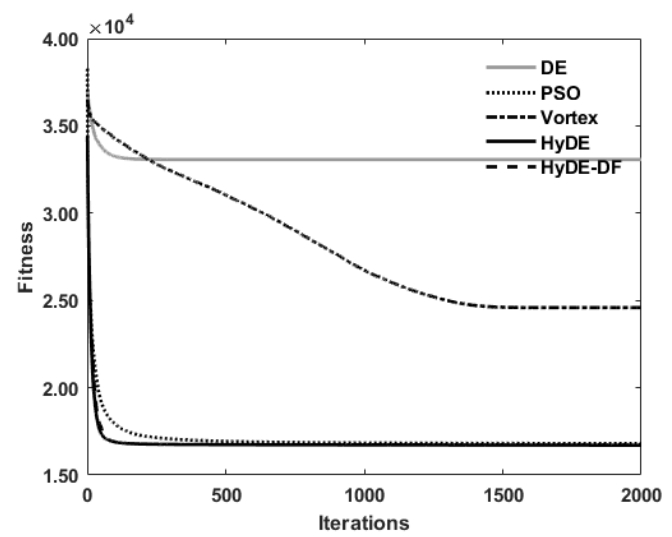

Fig. 5: Average convergence considering demand flexibility.

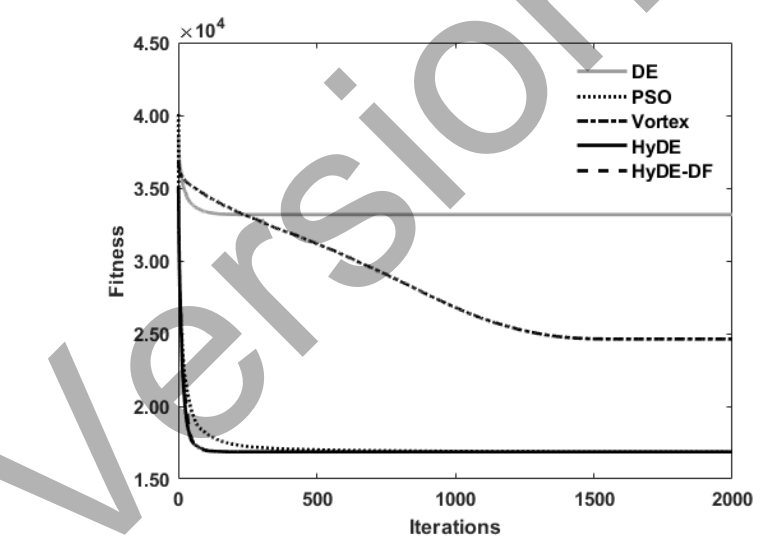

Fig. 6: Average convergence not considering demand flexibility.

and HyDE-DF combine different characteristics (observed and demonstrated in other studies), which give them the ability of self-tuning of parameters, and fast convergence capabilities (exploitation) in the first phase of the evolutionary process, while gradually switching to a local search phase (exploration) in the final steps of the evolutionary process. Due to best overall cost obtained by these two approaches the authors took one of them to show the ERM results. The HyDE-DF algorithm considering demand flexibility was chosen.

The energy resource management results (in terms of generation) is presented in Fig. 7. The total scheduled energy resources are 179.75 MWh. For the total external supplier acquisition (main), the amount is $109.57 \mathrm{MWh}$, while PV, Wind, CHP, and Small Hydro are 12.21 MWh, 26.23 MWh, 24.93 MWh and 1.10 MWh, respectively. Regarding Fig. 8 it presents the energy resource management results (in terms of consumption). The total ESS discharge is $0.00105 \mathrm{MWh}$, the EV discharge is $0.106 \mathrm{MWh}$, while the total scheduled demand flexibilty is $5.59 \mathrm{MWh}$. The total market purchase, the nonsupplied demand and the generation curtailment power are 0 MWh.The results for ESS charge, market sale, EV charge and power losses are 0.0051 MWh, 14.14 MWh, 8.24 MWh, and $0.2716 \mathrm{MWh}$, respectively. 


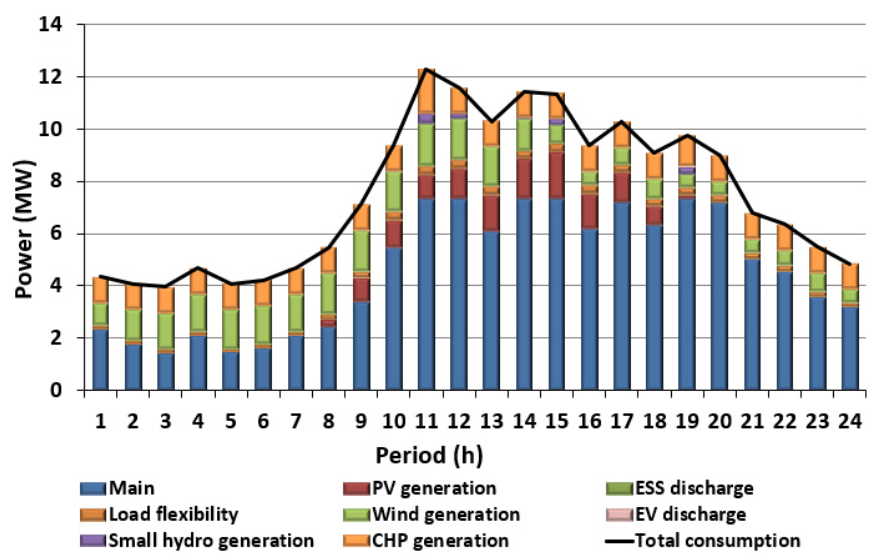

Fig. 7: Energy resource management - generation.

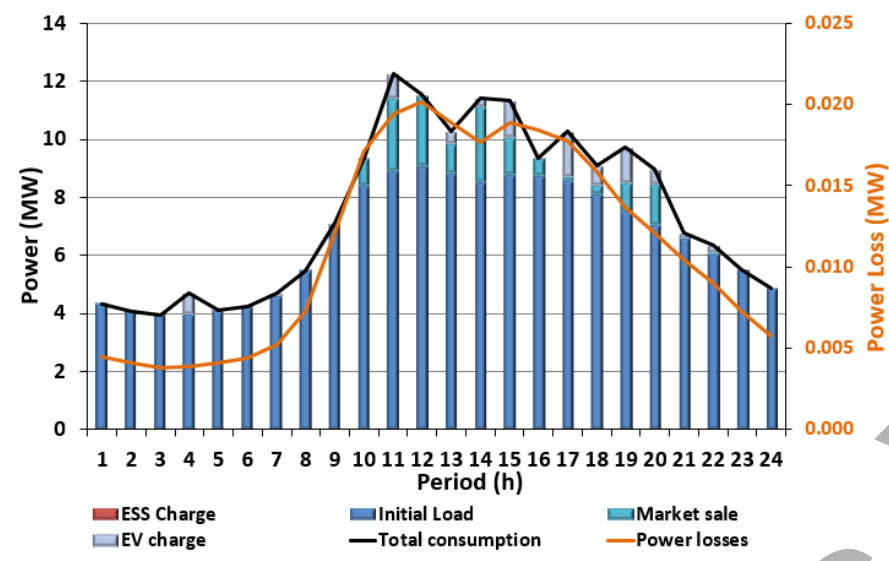

Fig. 8: Energy resource management - consumption.

\section{CONCLUSIONS}

This paper discusses the application of evolutionary algorithms to solve the large-scale energy resource management problem of aggregators in a smart grid. This problem is a combinatorial problem of large-scale nature when many distributed energy resources are considered. Due to non-linearity features of the problem, it is usually classified as MINLP. MINLP techniques require significant computer resources. To mitigate this, the evolutionary algorithm can play an important role. For this propose, a comparison between five evolutionary algorithms to solve the energy resource management problem was performed. The results suggest that the two variants of differential evolutionary algorithm, HyDE and HyDE-DF, can provide better results for the aggregator, i.e. lower operation costs (with a small advantage for HyDE - around 15 m.u.). It was also verified the advantage for the aggregator of using demand flexibility, reaching $0.95 \%$ and $0.86 \%$ of operation cost improvement with HyDE and HyDE-DF algorithms, respectively. As future work, the model will be adapted to include a competitive environment, where exist several aggregators and different business models for distribution system operator considering network technical constraints independent from the aggregators.

\section{REFERENCES}

[1] Shi You, Chresten Traeholt, and Bjarne Poulsen. A market-based Virtual Power Plant. In 2009 International Conference on Clean Electrical Power, pages 460-465. IEEE, 62009.

[2] Reza Hemmati, Hedayat Saboori, and Saeid Saboori. Assessing wind uncertainty impact on short term operation scheduling of coordinated energy storage systems and thermal units. Renewable Energy, 95:7484, 92016.

[3] Xi Fang, Satyajayant Misra, Guoliang Xue, and Dejun Yang. Smart Grid - The New and Improved Power Grid: A Survey. IEEE Communications Surveys \& Tutorials, 14(4):944-980, 2012.

[4] Daywes Pinheiro Neto, Elder Geraldo Domingues, António Paulo Coimbra, Aníbal Traça de Almeida, Aylton José Alves, and Wesley Pacheco Calixto. Portfolio optimization of renewable energy assets: Hydro, wind, and photovoltaic energy in the regulated market in Brazil. Energy Economics, 64:238-250, 52017.

[5] Pedro Faria, Joao Spinola, and Zita Vale. Aggregation and Remuneration of Electricity Consumers and Producers for the Definition of DemandResponse Programs. IEEE Transactions on Industrial Informatics, 12(3):952-961, 62016.

[6] Kang Miao Tan, Vigna K. Ramachandaramurthy, and Jia Ying Yong. Integration of electric vehicles in smart grid: A review on vehicle to grid technologies and optimization techniques. Renewable and Sustainable Energy Reviews, 53:720-732, 12016.

[7] Bin Zhou, Wentao Li, Ka Wing Chan, Yijia Cao, Yonghong Kuang, Xi Liu, and Xiong Wang. Smart home energy management systems: Concept, configurations, and scheduling strategies. Renewable and Sustainable Energy Reviews, 61:30-40, 82016.

[8] European commisssion. Accelerating Clean Energy in Buildings. Technical report, 2016.

[9] Thomas Strasser, Filip Andren, Johannes Kathan, Carlo Cecati, Concettina Buccella, Pierluigi Siano, Paulo Leitao, Gulnara Zhabelova, Valeriy Vyatkin, Pavel Vrba, and Vladimir Marik. A Review of Architectures and Concepts for Intelligence in Future Electric Energy Systems. IEEE Transactions on Industrial Electronics, 62(4):24242438, 42015 .

[10] João Soares, Tiago Pinto, Fernando Lezama, and Hugo Morais. Survey on Complex Optimization and Simulation for the New Power Systems Paradigm. Complexity, 2018:1-32, 82018.

[11] Swagatam Das, Ajith Abraham, Uday K. Chakraborty, and Amit Konar. Differential Evolution Using a Neighborhood-Based Mutation Operator. IEEE Transactions on Evolutionary Computation, 13(3):526-553, 2009.

[12] Fernando Lezama, Joao Soares, Ricardo Faia, Tiago Pinto, and Zita Vale. A New Hybrid-Adaptive Differential Evolution for a Smart Grid Application Under Uncertainty. In 2018 IEEE Congress on Evolutionary Computation (CEC), pages 1-8. IEEE, 72018.

[13] Swagatam Das and Ponnuthurai Nagaratnam Suganthan. Differential Evolution: A Survey of the State-of-the-Art. IEEE Transactions on Evolutionary Computation, 15(1):4-31, 22011.

[14] João Soares, Mohammad Ali Fotouhi Ghazvini, Marco Silva, and Zita Vale. Multi-dimensional signaling method for population-based metaheuristics: Solving the large-scale scheduling problem in smart grids. Swarm and Evolutionary Computation, 29:13-32, 82016.

[15] Berat Doğan and Tamer Ölmez. A new metaheuristic for numerical function optimization: Vortex Search algorithm. Information Sciences, 293:125-145, 22015.

[16] I.J. Ramirez-Rosado and J.L. Bernal-Agustin. Genetic algorithms applied to the design of large power distribution systems. IEEE Transactions on Power Systems, 13(2):696-703, 51998.

[17] Energy Information Administration. Levelized Cost and Levelized Avoided Cost of New Generation Resources in the Annual Energy Outlook 2019. Technical report, 2019.

[18] Kenneth Price, Rainer M Storn, and Jouni A Lampinen. Differential Evolution. Springer-Verlag, Berlin/Heidelberg, 2006.

[19] Fernando Lezama, João Soares, Ricardo Faia, and Zita Vale. Hybridadaptive differential evolution with decay function (HyDE-DF) applied to the 100-digit challenge competition on single objective numerical optimization. In Proceedings of the Genetic and Evolutionary Computation Conference Companion on - GECCO '19, pages 7-8, New York, New York, USA, 2019. ACM Press.

[20] D. Thukaram, H.M. Wijekoon Banda, and Jovitha Jerome. A robust three phase power flow algorithm for radial distribution systems. Electric Power Systems Research, 50(3):227-236, 61999. 